\title{
Predictive value of NLR, TILs (CD4+/CD8+) and PD-L1 expression for prognosis and response to preoperative chemotherapy in gastric cancer
}

\author{
Ina Valeria Zurlo ${ }^{1} \cdot$ Mattia Schino ${ }^{2} \cdot$ Antonia Strippoli $^{1} \cdot$ Maria Alessandra Calegari $^{1} \cdot$ Alessandra Cocomazzi $^{2}$. \\ Alessandra Cassano ${ }^{1,3} \cdot$ Carmelo Pozzo $^{1,3}$. Mariantonietta Di Salvatore ${ }^{1} \cdot$ Riccardo Ricci $^{1,2}$. Carlo Barone ${ }^{1,3}$. \\ Emilio Bria ${ }^{1,3} \cdot$ Giampaolo Tortora ${ }^{1,3} \cdot$ Luigi Maria Larocca $\cdot$ Michele Basso ${ }^{1} \cdot$ Maurizio Martini ${ }^{1,2}$ (1)
}

Received: 4 January 2021 / Accepted: 6 May 2021 / Published online: 19 May 2021

(c) The Author(s) 2021

\begin{abstract}
The combination of perioperative chemotherapy plus complete surgical resection is currently accounted as the first-choice strategy in patients with locally advanced Gastric Cancer (LAGC). Nevertheless, the partial response rate makes it necessary to search biological parameters useful to select patients who would benefit most from neoadjuvant chemotherapy (NAD-CT). We performed a retrospective analysis on a cohort of 65 LAGC cases, EBV negative and without MMR defect, submitted to perioperative chemotherapy plus surgical resection. We evaluated the neutrophil-lymphocytes ratio (NLR) in peripheral blood, the TILs density (reported as CD4/CD8 tissue ratio) and PD-L1 expression by immunohistochemistry on bioptic tissues before the treatment. Results were correlated with the biological features, histological response (TRG) and clinical outcome (PFS and OS). We found that NLR, TILs and PD-L1 expression showed a significant correlation with TNM stage, lymphovascular invasion and response to NAD-CT (TRG). Correlating the NLR, TILs and PD-L1 expression with PFS and OS, we found that patients with lower NLR levels ( $<2.5$ ratio), lower TILs ( $<0.2$ ratio) and higher PD-L1 level $(C P S \geq 1)$ had a significantly better PFS and OS than those with higher NLR, higher TILs and lower PD-L1 expression $(p<0.0001)$. Multivariate and multiple regression analyses confirmed the predictive and prognostic role of all three parameters, especially when all three parameters are combined. Our study demonstrated that pre-treatment NLR, TILs and PD-L1 expression are predictive and prognostic parameters in NAD-CT-treated LAGC suggesting a pivotal role of the systemic and tumor microenvironment immunological profile in the response to chemotherapy.
\end{abstract}

Keywords Gastric cancer $\cdot$ Neoadjuvant chemotherapy $\cdot$ Predictive factors $\cdot$ Precision medicine $\cdot$ Immunological status

$\begin{array}{ll}\text { Abbreviations } \\ \text { ALC } & \text { Absolute lymphocytes count } \\ \text { ANC } & \text { Absolute neutrophil count } \\ \text { CPS } & \text { Combined positive score } \\ \text { EBV } & \text { Epstein-Barr virus } \\ \text { GC } & \text { Gastric cancer } \\ \text { IHC } & \text { Immunohistochemistry }\end{array}$

Ina Valeria Zurlo and Mattia Schino have contributed equally to this work.

Michele Basso and Maurizio Martini have shared senior authorship.

Maurizio Martini

maurizio.martini@unicatt.it

Extended author information available on the last page of the article
LACG Locally advanced gastric cancer

MSI Microsatellite instability

MMR Mismatch repair

NAD-CT Neoadjuvant chemotherapy

NLR Neutrophil-lymphocytes ratio

OS Overall survival

PFS Progression free survival

TIL Tumor-infiltrating lymphocytes

TME Tumor microenvironment

TRG Tumor regression grade

\section{Background}

Gastric cancer (GC) stands as the fifth most frequently diagnosed malignancy and the third leading cause of cancer death [1]; in Western countries, about two-thirds of GC patients are 
diagnosed with locally advanced cancer (LAGC) or metastatic disease [2].

The combination of perioperative chemotherapy plus complete surgical resection (R0) is currently considered as the firstchoice strategy to improve progression-free survival (PFS) and overall survival (OS) in LAGC patients [3-5].

Nevertheless, while it is true that latest years brought to significant advances both in surgery and combined drug regimens, the overall response rate to chemotherapy is still less than 50\%, keeping the prognosis rather dismal [3-5]. Therefore, it would be advantageous to select patients who would benefit most from neoadjuvant chemotherapy (NAD-CT) and who might gain the best survival advantage $[6,7]$.

In this way, several studies have highlighted that tumor immune infiltrations [7-9], by defining neoplasms either as immunologically "cold," when they exhibit a low level of tumor infiltrating lymphocytes (TILs), or as immunologically "hot," when TILs' level is high, could not only be a prognostic marker in GC in general terms-thus supporting the role of immunotherapy (IT) in GC itself [10, 11]—but could also play a role in predicting GC response to NAD-CT $[7,12,13]$. However, this topic is still debated, since current literature data are conflicting and ultimately leading to disagreement on both type and association of biomarkers to be used to investigate GC-related "immunological status" [6, 7, 12].

Recently, systemic immune-inflammation indexes, based on routinely measurable peripheral blood parameters - such as neutrophil-to-lymphocyte ratio (NLR) - have been proposed as manageable prognosticators and surrogates of cancerrelated inflammation in a variety of neoplasms [14], including $\mathrm{GC}$, also after NAD-CT treatment $[6,7,15]$.

Furthermore, lymphocyte sub-sets in GC have also been considered, hinting a significant correlation between TILs, expressed as CD4+/CD8+ T cells tissue ratio, and survival $[12,16,17]$, while other studies focused on high microsatellite instability, MSI-H and PD-L1 expression, as a potential indicator of resistance to NAD-CT [12, 18, 19].

Based on the latest evidence, we performed a retrospective monocentric analysis, in a cohort of NAD-CT treated locally advanced GCs, EBV negative and with normal expression of mismatch repair (MMR) proteins, to evaluate the baseline NRL, TILs density (reported as CD4+/CD8+ T cells tissue ratio) and PDL-1 expression, as indicators of the GC immunological status, and their correlation with the main clinical and biological features, including response to therapy.

\section{Methods}

\section{Patients' features}

This exploratory monocentric retrospective analysis was performed at the Unit of Medical Oncology and Unit of
Pathology of Fondazione Policlinico Universitario "Agostino Gemelli"-IRCCS, Università Cattolica del Sacro Cuore, Rome. Clinical and pathological records from 112 CT-naïve patients with LAGC treated with NAD-CT from January 2012 to January 2017 were reviewed. Inclusion and exclusion criteria, chemotherapy regimen, post-treatment follow-up examinations and other clinical parameters were reported in supplementary material. Finally, 65 patients who met the inclusion criteria were enrolled in this study, while 47 patients were excluded. The main clinical and biological characteristics of the cohort are shown in Table 1.

\section{Blood parameters}

Venous blood samples were taken at diagnosis, before NAD-CT, and four weeks or more after the last dose of chemotherapy and within 1 week before the surgical treatment. Hence, baseline or pre-treatment NLR (preNRL) and post-treatment NLR (post-NRL) were calculated dividing the absolute neutrophil count (ANC) by the absolute lymphocyte count (ALC; NLR $=\mathrm{ANC} / \mathrm{ALC}$ ). The cut-off values for white blood cells ANC $\left(>4000 / \mathrm{mm}^{3}\right.$ and $\left.\leq 4000 / \mathrm{mm}^{3}\right)$, ALC $\left(>1500 / \mathrm{mm}^{3}\right.$ and $\left.\leq 1500 / \mathrm{mm}^{3}\right)$ and NLR $(>2.5$ and $\leq 2.5)$ were defined considering the median values and data from previous studies $[6,7]$.

\section{HER2 gene amplification}

HER2 amplification was performed using the INFORM HER2/neu Dual ISH DNA Probe Cocktail assay (Ventana Medical Systems, Inc, Tucson, Arizona) as previously described (supplementary material) [20].

\section{TILs (CD4+/CD8+ T-cells ratio) evaluation and PD-L1 expression}

TILs were evaluated as the CD4+/CD8+ T-cells ratio. The expression of $\mathrm{CD} 4+$ and $\mathrm{CD} 8+$ was assessed by immunohistochemistry (IHC; supplementary material) as previously described with few modifications [8, 9, 21].

PD-L1 expression was evaluated using immunohistochemistry (IHC) and anti-PD-L1 rabbit monoclonal antibody (PD-L1 IHC 22C3 pharmDX; Agilent Technolologies, Carpinteria, CA, USA). Detailed methods are reported in supplementary material. Samples were considered PD-L1 negative if CPS was less than 1 and PD-L1 positive if CPS was 1 or more.

\section{Statistical analysis}

The objective of this analysis was to explore the correlation between clinical and biological parameter, TILs density (reported as CD4/CD8 tissue ratio), PDL-1 expression 
Table 1 Patient characteristics

\begin{tabular}{|c|c|}
\hline & $n=65$ \\
\hline Age, mean $( \pm S D)$ & $63(9.3)$ \\
\hline \multicolumn{2}{|l|}{ Gender $[n(\%)]$} \\
\hline Male & $41(63.1)$ \\
\hline Female & $24(36.9)$ \\
\hline \multicolumn{2}{|l|}{ TNM stage* $[n(\%)]$} \\
\hline IIIB & $35(53.85)$ \\
\hline IIIC & $30(46.15)$ \\
\hline \multicolumn{2}{|l|}{ Tumor site $[n(\%)]$} \\
\hline Upper & $25(38.5)$ \\
\hline Middle & $18(27.7)$ \\
\hline Lower & $22(33.8)$ \\
\hline \multicolumn{2}{|l|}{ Histological subtype $[n(\%)]$} \\
\hline Intestinal & $31(47.7)$ \\
\hline Diffuse & $34(52.3)$ \\
\hline \multicolumn{2}{|l|}{ HER2 status $[n(\%)]$} \\
\hline HER2+ & $15(23.1)$ \\
\hline HER2- & $50(76.9)$ \\
\hline \multicolumn{2}{|c|}{ Response to NAD-CT [ $n(\%)]$} \\
\hline TRG 1-2 & $34(52.3)$ \\
\hline TRG 3-5 & $31(47.7)$ \\
\hline PFS, mean (months, SD) & $26.3(18.1)$ \\
\hline OS, mean (months, SD) & $34.6(18.7)$ \\
\hline \multicolumn{2}{|l|}{$\operatorname{LVI}[n(\%)]$} \\
\hline Yes & $31(47.7)$ \\
\hline No & $34(52.3)$ \\
\hline \multicolumn{2}{|l|}{ Perineural infiltration (\%) } \\
\hline Yes & $20(30.8)$ \\
\hline No & $45(69.2)$ \\
\hline \multicolumn{2}{|l|}{ NLR pre-chemotherapy (\%) } \\
\hline$\geq 2.5$ & $35(53.8)$ \\
\hline$<2.5$ & $30(46.2)$ \\
\hline \multicolumn{2}{|c|}{ NLR post-chemotherapy (\%) } \\
\hline$\geq 2.5$ & $29(44.6)$ \\
\hline$<2.5$ & $36(55.4)$ \\
\hline \multicolumn{2}{|l|}{$\mathrm{CD} 4+/ \mathrm{CD} 8+$ ratio $(\%)$} \\
\hline$\geq 0.2$ & $32(49.2)$ \\
\hline$<0.2$ & $33(50.8)$ \\
\hline \multicolumn{2}{|l|}{ PD-L1 expression, CPS (\%) } \\
\hline$<1$ & $34(52.3)$ \\
\hline$\geq 1$ & $31(47.7)$ \\
\hline
\end{tabular}

* According to the 8th edition of the American Joint Committee on Cancer (AJCC) guidelines. SD, standard deviation. PFS, progressionfree survival. OS, overall survival. LVI, lympho-vascular invasion.

and NLR, in a cohort of patients with LAGC treated with NAD-CT.

Statistical analysis was performed using GraphPadPrism 5 software (Graph Pad Software, San Diego, CA) and MedCalc version 10.2.0.0 (MedCalc Software, Mariakerke, Belgium; supplementary material) [20, 22].

The evaluation of the tumor response to neoadjuvant treatment was performed using Mandard's classification system (Tumor Regression Grade, TRG) [23]. Responders were defined as TRG 1-2, and non-responders were defined as TRG 3-5 [24]. Progression Free Survival (PFS) and Overall Survival (OS) were the survival endpoints. PFS was calculated as the time from NAD-CT beginning to any evidence of disease progression (either local/regional relapses or distant metastases) or death, whichever occurred first. OS was calculated as the time from NAD-CT beginning to the patient's death, due to any cause.

\section{Results}

\section{Patient characteristics and treatment response}

Main clinicopathologic characteristics of our cohort (65 consecutive patients with LAGC treated with NAD-CT) are reported in Table 1.

Mean age at the time of diagnosis was 63 years, and 63\% of patients were male. Clinical TNM stage was IIIB in 35 cases (53.85\%) and stage IIIC in 30 cases (46.15\%), respectively. Twenty-five patients out of 65 (38.5\%) had an upper site located GC (Siewert type 2 or 3), 18 out of 65 (27.7\%) had a middle site located GC, and 22 out of $65(33.8 \%)$ a lower site GC. Histologically, 31 out of $65(47.7 \%)$ had an intestinal subtype GC, while 34 out of 65 (52.3\%) a diffuse subtype GC. HER2 amplification was found in 15 out of 65 cases $(23.1 \%)$, while 50 patients $(76.9 \%)$ were negative. The post- NAD-CT response was evaluated according to Mandard-TRG pathological response system: 34 out of 65 patients (55.4\%) had a good tumor regression (TRG 1-2), while 29 out of 65 (44.6\%) had a poor tumor regression (TRG 3-5).

\section{Blood neutrophil-lymphocyte ratio}

Mean pre-chemotherapy neutrophil and lymphocyte counts were 4940 and 1801 per $\mathrm{mm}^{3}$, respectively. Mean pre-chemotherapy NLR was 3.5 (range $0.57-3.8$ ); by considering 2.5 as the cut-off value for NLR, as reported in the literature data [6], we found that 30 out of 65 patients (46.2\%) had a low NLR ratio, while 35 patients (53.8\%) had a high NLR ratio (Table 1). On the contrary, mean postchemotherapy neutrophil and lymphocyte counts were 4010 and 1460 per $\mathrm{mm}^{3}$, respectively. Mean post-chemotherapy NLR was 2.74 (range $0.72-12.6$ ); by considering 2.5 as the cut-off value for NLR, we found that 36 out of 65 patients (55.4\%) had a low NLR ratio, while 29 patients (44.6\%) had a high NLR ratio (Table 1). Correlating the ANC and ALC, in the pre-treatment blood samples, with the clinical 
and biological parameters of our cohort, we found that male gender was significantly correlated with the ANC $(p<0.001$; Table 1S), while all other parameters did not have any significant association, either with ANC or with ALC (Table 1S). Moreover, NLR in pre-treatment blood samples showed a significant correlation with TNM stage $(p=0.013)$, lymphovascular invasion (LVI, $p<0.001)$ and TRG ( $p=0.001$; Table 2). Twenty-four patients with a low baseline NLR level remained in this group after first-line chemotherapy (Table 3). By contrast, 6 patients from this group were transferred to the high NLR level group after NAD-CT. Twenty-three patients with a high baseline NLR level retained this level after first-line chemotherapy. By contrast, 12 patients with a high baseline NLR level were transferred to the low NLR level group after NAD-CT. When we correlated the changing in the NLR level with the tumor response to neoadjuvant treatment (TRG), we found that patients who remained in or were transferred to the low NLR level subgroup following NAD-CT exhibited improved response, compared to patients who remained in or were transferred to the high NLR level group (Table 3). Interestingly, we noted a significant decrement in the NRL values between pre- and post-chemotherapy ( $p=0.0033$, paired $t$-test; Fig. 1S, panel A).

\section{Immunohistochemistry for TILs (CD4+/CD8+ T cells ratio) in GC tissue}

Mean pre-chemotherapy CD4+/CD8+ T cells tissue ratio (TILs) was 0.2 (range from 0.03 to 5.53, Fig. 1). Using the TILs' mean value as the cut-off value, we found that 33 out of 65 patients $(50.9 \%)$ had a low TILs, while 32 patients (49.2\%) had a high TILs (Table 1). Correlating TILs with the clinical and biological parameters of our cohort, we found that TNM stage $(p=0.025), \operatorname{LVI}(p<0.001)$ and TRG $(p=0.003)$ were significantly correlated with TILs (Table 2$)$, while none of the other parameters showed any significant association (Table 2). Interestingly, we found that TILs had a direct and significant correlation with pre-treatment NLR value (Spearman $r=0.6338 ; p<0.0001$; Fig. 2 S, panel A).

\section{Evaluation of PD-L1 expression (CPS)}

We found that 34 out of 65 patients (52.3\%) had a low PD-L1 expression $(\mathrm{CPS}<1)$, while 31 patients $(47.7 \%)$ had a high PD-L1 level (CPS $\geq 1$; Table 1 and Fig. 1). Correlating the PD-L1 expression with the clinical and biological parameters of our cohort, we found that LVI $(p<0.001$; Table 2), stage (IIIC vs IIIB; $p=0.006$; Table 2$)$ and TRG $(p<0.001$; Table 2) were significantly correlated with PD-L1 level, while none of other parameters showed any significant association (Table 2). We also found that PD-L1 expression had an indirect and significant correlation with both pre-treatment NLR value (Spearman $r=-0.781 ; p<0.0001$; Fig. 2S, panel B) and TILs (Spearman $r=-0.567$; $p<0.0001$; Fig. 2S, panel C).

\section{Prognostic variables for PFS and OS}

Mean PFS and mean OS were 26.3 months and 34.6 months, respectively. When we correlate the pre-treatment NLR with PFS and OS, we found that patients with lower NLR levels had a significantly better PFS and OS than those with higher NLR levels (Fig. 2 panel A for PFS: median PFS for lower pre-treatment NLR level 66 months versus median PFS for higher pretreatment NLR level 29 months, $p<0.0001$, HR 7.21, 95\% CI from 3.03 to 17.12; Fig. 3 panel A for OS: median OS for lower pre-treatment NLR level 73 months versus median OS for higher pre-treatment NLR level 37 months, $p<0.0001$, HR 6.60, 95\% CI from 2.86 to 15.23 ). A similar but lower significant result was observed correlating the post-treatment NLR with PFS and OS (Fig. 1S panel B for PFS: median PFS for lower post-treatment NLR level 61 months versus median PFS for higher post-treatment NLR level 27 months, $p=0.001$, HR $4.20,95 \%$ CI from 1.78 to 9.91; Fig. 1S panel C for OS: median OS for lower posttreatment NLR level 64 months versus median OS for higher post-treatment NLR level 37 months, $p=0.015$, HR 2.79, 95\% CI from 1.22 to 6.42 ).

Patients with lower TILs had a significant association with better PFS and OS than those with higher CD4+/ CD8+ T-cell tissue ratio (Fig. 2 panel B for PFS: median PFS for patients with lower CD4+/CD8+ T cells ratio 65 months versus median PFS for patients with higher CD4+/ CD8+ T cell ratio patients 18 months, $p<0.0001$, HR 11.88 , 95\% CI from 4.65 to 30.33 ; Fig. 3 panel B for OS: median OS for patients with lower CD4+/CD8+ T cell ratio 75 months versus median OS for patients with higher CD4+/ CD $8+$ T cell ratio 27 months, $p<0.0001$, HR $11.57,95 \% \mathrm{CI}$ from 4.76 to 28.12).

Moreover, higher PD-L1 level (CPS $\geq 1$ ) in pretreated GC tissue was significantly associated with a better PFS and OS in comparison with those with lower PD-L1 score (CPS $<1$; Fig. 2 panel C for PFS: median PFS for PD-L1 CPS $\geq 1$ patients 20 months versus median PFS for PD-L1 CPS $<1$ patients 67 months, $p<0.0001$, HR $0.09924,95 \%$ CI from 0.04029 to 0.2445 ; Fig. 3 panel C for OS: median OS for PD-L1 CPS $\geq 1$ patients 28 months versus median OS for PD-L1 CPS $<1$ patients 74 months, $p<0.0001$, HR 0.1154, $95 \%$ CI from 0.04865 to 0.2737 ).

In addition, Kaplan-Meir analysis showed that variables predicting improved PFS and OS were TNM-IIIB (Table 2S, PFS, $p=0.0379$; OS, $p=0.0174$ ), absence of LVI (Table 2S, PFS, $p=0.0515$; OS, $p=0.0196$ ) and TRG $1-2$ (Table 2S, PFS, $p=0.0244$; OS, $p=0.0112$ ). No correlation was found 


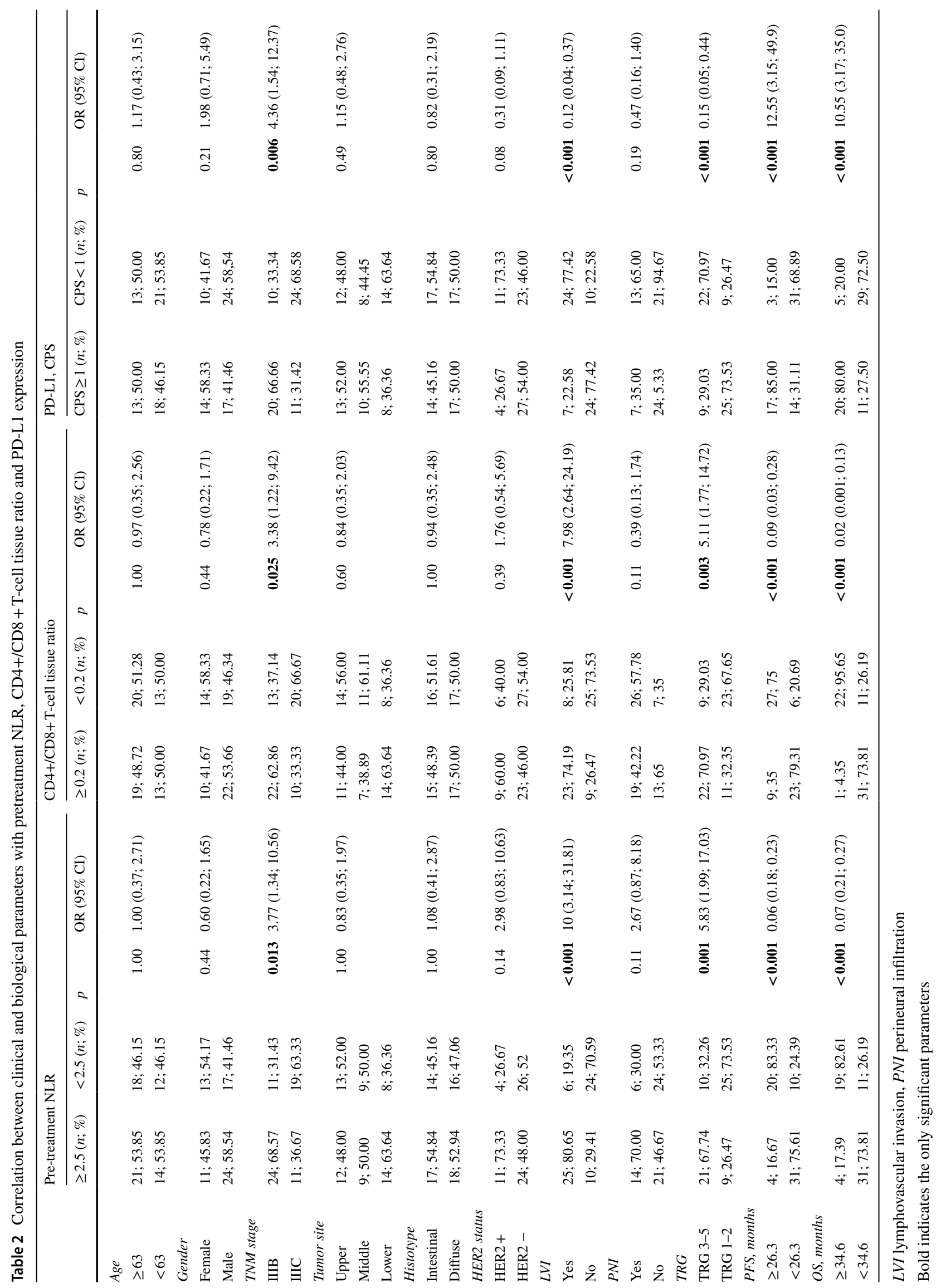


Table 3 Relationship between changes in the NLR level and tumor response to neoadjuvant treatment (TRG)

\begin{tabular}{lllllc}
\hline Pre-chemotherapy & Post-chemotherapy & $\begin{array}{l}\text { TRG 1-2 } \\
(n=34)\end{array}$ & $\begin{array}{l}\text { TRG 3-5 } \\
(n=31)\end{array}$ & OR & $p$ value \\
\hline NLR $<2.5(n=30)$ & NLR $<2.5(n=24)$ & 15 & 9 & 21.21 & 0.017 \\
& NLR $\geq 2.5(n=6)$ & 0 & 6 & & \\
NLR $\geq 2.5(n=35)$ & NLR $<2.5(n=12)$ & 11 & 1 & 20.63 & 0.002 \\
& NLR $\geq 2.5(n=23)$ & 8 & 15 & & \\
\hline
\end{tabular}

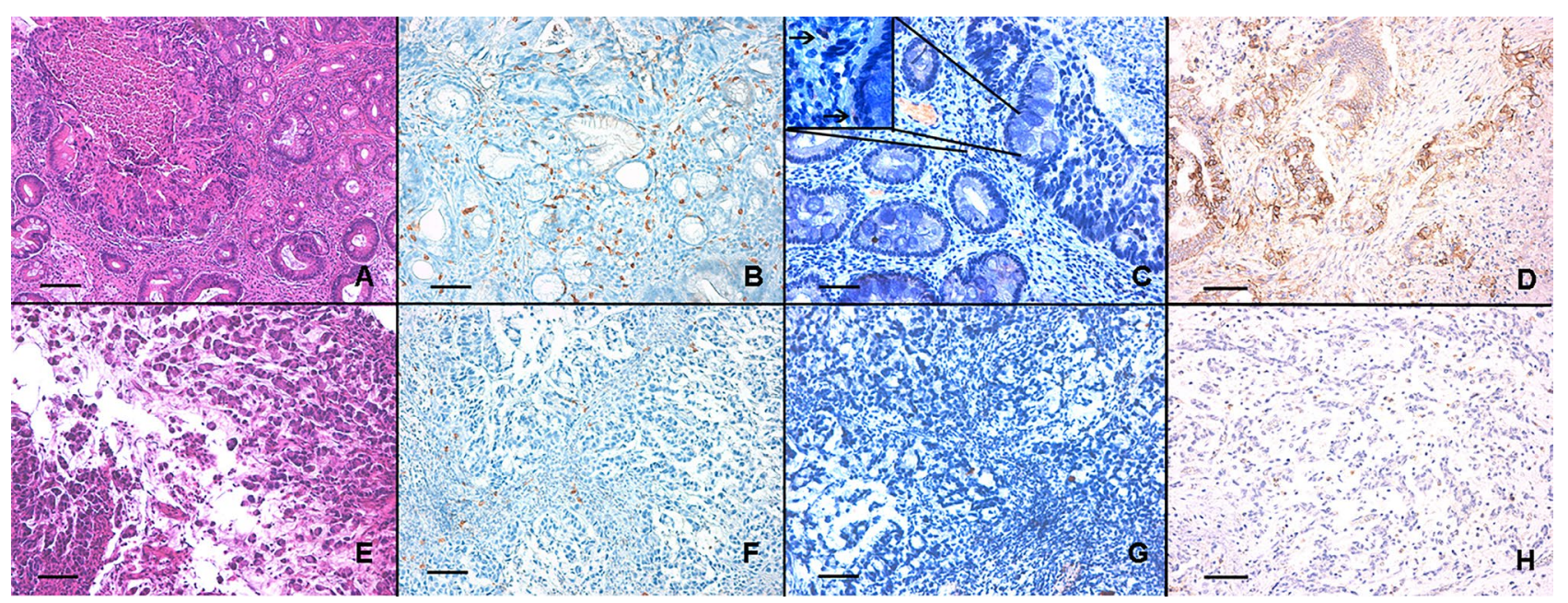

Fig. 1 The figure shows two LAGC samples of intestinal and diffuse histotype cancer (panel A and E, respectively, E\&E, $\times 200$ magnification, bare scale $150 \mathrm{~mm}$ ); the $\mathrm{CD} 4+$ and $\mathrm{CD} 8+$ cell counts (figure $\mathrm{B}$ and $\mathrm{F}$ for $\mathrm{CD} 8+$ cells; figure $\mathrm{C}$ and $\mathrm{G}$ for $\mathrm{CD} 4+$ cells; $\times 200$ magni- fication, bare scale $150 \mathrm{~mm}$; the box in the panel $\mathrm{C}$ shows a detail of the image at $\times 400$ magnification, where the arrows indicate the CD4 + cells); the PD-L1 expression, evaluated as CPS score (figure $\mathrm{D}, \mathrm{CPS} \geq 1$ and $\mathrm{H}, \mathrm{CPS}<1 ; \times 200$ magnification, bare scale $150 \mathrm{~mm}$ )

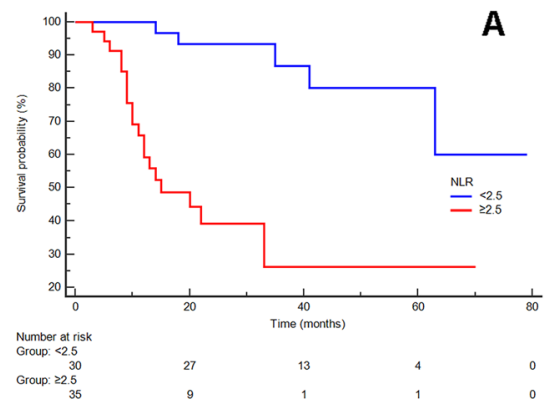

Fig. 2 Kaplan-Meier curves for PFS of NAD-CT-treated LAGC patients stratified by pre-treatment NLR (panel A), CD4+/CD8+ T cells tissue ratio (TILs; panel B) and PD-L1 expression (evaluated as CPS score; panel C). Patients with lower NLR $<2.5$, TILs $<0.2$ and with PD-L1 CPS $\geq 1$ (blue-line) were significantly associated with a better PFS $(p<0.0001)$ respect to those patients with NLR $\geq 2.5$, TILs $\geq 0.2$ and with PD-L1 CPS $<1$ (red-line) with gender, age, tumor site, histological subtypes, HER2 expression nor perineural infiltration (Table 2S).

Multivariate analysis of PFS including pre-treatment NLR, TILs and PD-L1 expression, stage, histological subtypes, TRG and lymphovascular invasion showed that NLR, TILs, PD-L1 expression before NAD-CT were significant predictors (Table $4 ; p=0.0001$ for TILs; $p=0.0013$ for PD-L1 expression; $p=0.0036$ for pretreatment NLR). Similarly, multivariate analysis of OS, including pre-treatment NLR, TILs and PD-L1 expression, stage, histological subtypes, TRG and lymphovascular invasion, showed that the independent prognostic variables were pretreatment NLR, TILs and PD-L1 expression (Table 4; 

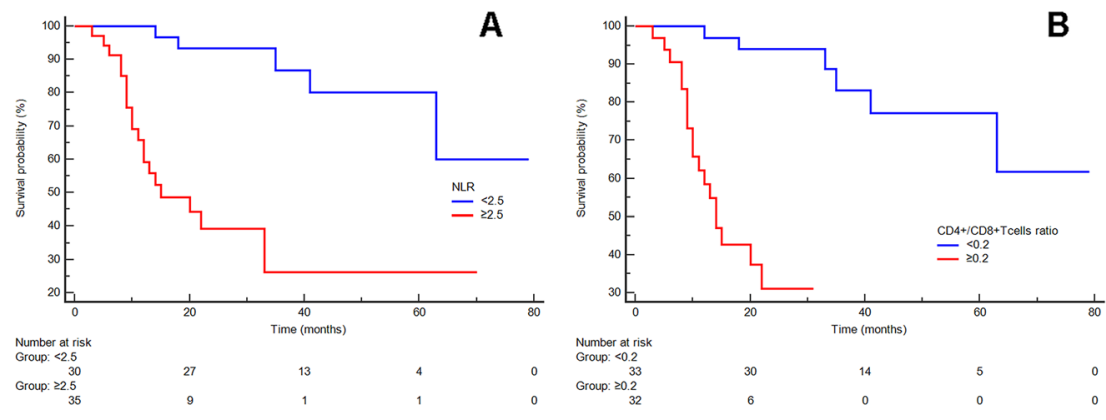

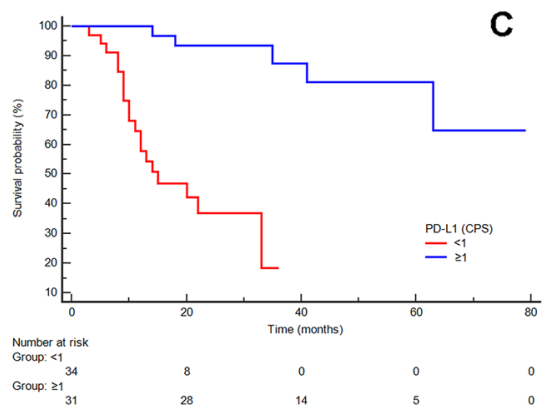

Fig. 3 Kaplan-Meier curves for OS of NAD-CT-treated LAGC patients stratified by pre-treatment NLR (panel A), CD4+/CD8+T cells tissue ratio (TILs; panel B) and PD-L1 expression (evaluated as CPS score; panel C). Patients with lower NLR $<2.5$, TILs $<0.2$ and with PD-L1 CPS $\geq 1$ (blue-line) were significantly associated with a better OS $(p<0.0001)$ respect to those patients with $N L R \geq 2.5$, TILs $\geq 0.2$ and with PD-L1 CPS $<1$ (red-line)
Table 4 Multivariate analysis for PFS and OS

\begin{tabular}{lllllll}
\hline & $b$ & SE & Wald & $p$ & $\operatorname{Exp}(b)$ & 95\% CI of $\operatorname{Exp}(b)$ \\
\hline Covariate for PFS & & & & & & \\
CD4+/CD8+ ratio & 2.7528 & 0.7025 & 15.3540 & 0.0001 & 15.6868 & $3.9584-62.1645$ \\
PD-L1 (CPS) & 2.1720 & 0.6765 & 10.3092 & 0.0013 & 0.1139 & $0.0303-0.4291$ \\
Pre-treatment NLR & 1.8501 & 0.6357 & 8.4692 & 0.0036 & 6.3605 & $1.8295-22.1123$ \\
Covariate for OS & & & & & & \\
CD4+/CD8+ ratio & 2.8712 & 0.8072 & 12.6524 & 0.0004 & 17.6577 & $3.6295-85.9058$ \\
PD-L1 (CPS) & 2.5776 & 0.7249 & 12.6431 & 0.0004 & 0.0760 & $0.0183-0.3145$ \\
Pre-treatment NLR & 2.0307 & 0.6266 & 10.5042 & 0.0012 & 7.6195 & $2.2314-26.0178$ \\
\hline
\end{tabular}

$b=$ coefficient estimates; $\mathrm{SE}=$ standard error for coefficient estimates $b ; \operatorname{Exp}(b)=$ Hazard Ratio value; 95\% CI of $\operatorname{Exp}(b)=95 \%$ confidence interval of Hazard Ratio $p=0.0004$ for both TILs and PD-L1 expression; $p=0.0012$ for pretreatment NLR).

The predictive value for PFS and OS of each parameter (pre-treatment NLR, PD-L1 expression and TILs) was evaluated, first individually, then combined (two or three parameters), performing a multiple regression analysis. The predictive value was significantly higher only when the three parameters were considered jointly combined $(p<0.0001$ for both PFS and OS; r partial 0.7329 for PFS and $r$ partial 0. 6157 for OS).

\section{Discussion}

This monocentric study retrospectively investigated the relationship between systemic and tumor microenvironment (TME) immunological profile in patients with LAGC before receiving NAD-CT and their clinicopathologic outcome to identify some parameters which could help in selecting patients who might respond to $\mathrm{CT}$.

According to recent evidence $[6,7,15]$, we demonstrated low NLR in the peripheral blood of pre-treated NAD-CT LAGC was significantly associated with a favorable PFS and OS (both $p<0.0001$ ), also finding a significant association between low NLR and TRG $(p=0.001)$ and TNM stage $(p=0.013)$ and LVI $(p \leq 0.001)$. At the same time, we evaluated the peritumoral microenvironment, where the immune host cells (mainly lymphocytes) strictly interacted with neoplasm, demonstrating, in agreement with other authors [12, $16,17]$, the significant association with low CD4+/CD8+ T cell ratio with PFS, OS (both $p<0.001$ ), and the pathological response to NAD-CT (as TRG) in our cohort ( $p=0.003$ ). Similarly, investigating the expression of PD-L1, we also found, for the first time, that higher level of PD-L1 is associated with better PFS and OS (both $p<0.001$ ) and response to neoadjuvant therapy in LAGC $(p<0.001)$. In addition, higher level of CPS (CPS $\geq 1$ ) also had significant association with TNM stage $(p=0.006)$, TRG $(p<0.001)$ and LVI $(p \leq 0.001)$. Lastly, we found that post-treatment NLR levels were consistent with chemotherapeutic efficacy and clinical outcome, suggesting NLR levels following treatment, though less significantly than pre-treatment NRL, may also provide valuable prognostic and predictive information.

Several studies highlighted that the tumor immune status plays a role also in response to neoadjuvant radio-chemotherapy treatments in several human cancers $[6-8,12,17$, 
18, 25, 26]. Accordingly, considering the need to identify and select those patients who would benefit most from a neoadjuvant treatment, recent studies have tried to use different immune parameters as indicators of response to neoadjuvant treatments, sometimes finding inconsistent results, especially regarding the role of $\mathrm{T}$ cells subtypes [8-12, 17, $18,27]$. This probably depends on different factors such as the inhomogeneity of the analyzed cohorts, different neoadjuvant scheme treatments, as well as the use of different and single parameter to investigate the immune status which is inherently complex and variegated, and whose function is scarcely reducible to only one specific parameter.

Investigating three immunological prechemotherapy parameters, we found that the latter, besides being significantly correlated with better PFS and OS (both $p<0.005$ ) in univariate and multivariate analysis, had likewise a direct and significant correlation with each other. In fact, NLR, an expression of the immunosurveillance capacity of the host, the presence of higher levels of CD8 + in the tumor microenvironment, as an index of a patient's better immuneresponse, and PD-L1 expression, indicating the tumor's intrinsic immune-escape capability and consequently also the TME CD8 + T cells level, source of cytokines such as interferon gamma that induced the expression of PD-L1 [28, 29], mirrored some crucial aspects of the tumor/immunity interaction which, if considered together, better select LAGC patients who will benefit most from NAD-CT treatment. In fact, we demonstrated that joining the aforementioned parameters with one another makes stronger correlation between pre-chemotherapy immune status and clinical outcomes $(p<0.0001)$.

Although the chemotherapy tends to deeply modify the host immunity, often with detrimental and myelosuppressive effects, accumulating evidence indicates that the efficacy of conventional anticancer agents does not only involve direct cytostatic/cytotoxic effects, but also relies on the (re)activation of tumor-targeting immune responses, similarly to the abscopal effect due to the radiotherapy [30]. This chemotherapeutic effect might act in synergy with a most reactive, less depressive immune system and higher TME CD8 + [29], whose pre-chemotherapy status could be assessed with NLR, TILs and PD-L1 expression.

By ruling out both altered-MMRP carriers and EBV positive tumors, we managed to minimize confounding effects of other tumor variables on the outcomes and fluorouracil-based NAD-CT [18, 31]. Interestingly, PD-L1 expression, which was typically associated with MSI-H status and maybe indirectly to the fluorouracil-based NAD-CT outcome, as demonstrated in several tumors, showed here a significant correlation with PFS, OS and response to therapy only in LAGC patients displayed higher levels of this immune-suppressing protein. Probably, the upregulation of negative immune checkpoint proteins in gastric tumors is due to the tumor infiltration of effector T cells (especially the CD8 + cells), defined as "T cell inflamed phenotype," that in turn determine the upregulation of immune checkpoints, and not to a genomic instability [29]. This way, the PD-L1 target in gastric cancer could only be clinically effective (also in NAD-CT) for the subgroup of tumors that contain tumor-infiltrating immune cells and could explain the controversial results in the predictive effects of PD-L1 in response to PD-1/PD-L1 antibodies in GC [32].

Although over the last year, the standard NAD-CT in LAGC changed, after the publication of the FLOT4-trial data [33], we hypothesized that pre-treatment NLR, TILs and PD-L1 expression, even if requires demonstrating, could be a predictive and prognostic parameter also in this new fluorouracil-based regimen.

Unlike Wang et al. [34] who described a significant association between lower PD-L1 expression and HER2 gene amplification in GC, we did not find any significant association between this molecular feature and pre-treatment NLR, TILs and PD-L1 expression, suggesting that this alteration did not have a predictive role in NAD-CT-treated LAGC.

In addition, our data have shown that both the NRL levels before and after NAD-CT have a predictive and prognostic value in patients with LAGC. However, the post-treatment NRL shows less significance than the pre-treatment NRL. This could be partly explained by the bone morrow suppression of the NAD-CT and other biological parameters, as already reported by several authors $[9,35,36]$.

Heterogeneity in the immunohistochemical assessment for PD-L1 and TILs (CD4 and CD8) expression both within and between tumor sites is a well-documented phenomenon that could have important implications, especially for PD-L1 accuracy as a predictive biomarker [37-39]. In addition, other factors, such as the use of different antibodies, of different cut-off, of different immunohistochemical platforms and the inter-observer variability could play a role in the evaluation of these two parameters [37, 38]. Notwithstanding, the immunohistochemical assay for PD-L1 and TILs remains today one of the most used markers, especially for immunotherapy, and several studies have shown that it is possible to reduce the impact of the heterogeneity of expression in the evaluation of these two parameters. In this way, we perform the PD-L1 and TILs expression on baseline multisite sampling tumor tissue biopsies (median 4.02, with at least $50 \%$ tumor tissue), using the same antibodies and immunohistochemical platform, following standardized and well-defined conditions, and calculating the agreement indices (Cohen's K index) between the two pathologists (M.M. and R.R., who also went through a formal training program to evaluate CPS by the $22 \mathrm{C} 3$ pharmDx assay) in the evaluation of TILs and PD-L1 expression (that were very good: $k=0.82$ and $k=0.87$, respectively). 
Finally, although the evaluation of NRL, TIL and PD-L1 parameters can be an easily executable analysis and several studies have shown that these parameters, even if individually, could be useful in the selection of LAGC patients for NAD-CT; however, it appears necessary to better understand the molecular mechanisms underlying this association, before these being adopting in clinical practice. To this end, in-vitro models such as co-cultures of patient' immune cells and GC organoid could represent a valid model, in which different types of neoadjuvant treatment could be tested, also in combination with immunotherapy [40].

The main limitations of our study are the retrospective monocentric design and the relatively small though homogeneous-treated cohort of patients, so that our data need to be confirmed in other independent studies including NAD-CTtreated LAGC patients, and our immune parameters should be validated as a prognostic tool.

\section{Conclusions}

In summary, we propose that pre-treatment NLR, TILs and PD-L1 expression may be predictive and prognostic parameters in NAD-CT-treated LAGC suggesting a pivotal role of the tumor inflammatory microenvironment in the response to chemotherapy. These three parameters, as indicators of the patient tumor/immune system status, significantly correlated each other and could help the clinicians to recognize patients who might benefit from a NAD-CT with LAGC.

Supplementary Information The online version contains supplementary material available at https://doi.org/10.1007/s00262-021-02960-1.

Acknowledgements We thank Dr. Sara Capodimonti and Dr. Roberta Benvenuto for their technical support.

Authors' contribution IVZ, MS, MB and MM were the principal authors and the main contributors in writing the manuscript. AS, MAC, AC, AC and MDS analyzed and interpreted the patient data and reviewed the literature. MM, AC, RR and IVZ performed the immunohistochemistry analysis. EB, CB and GT read and corrected the manuscript. RR and LML corrected the English language errors. All authors read and approved the final manuscript.

Funding Open access funding provided by Università Cattolica del Sacro Cuore within the CRUI-CARE Agreement. This study was supported by Università Cattolica del Sacro Cuore, Fondi d'Ateneo, Linea D1 (2017 and 2018; MM).

Availability of data and materials The datasets used and/or analyzed during the current study are available from the corresponding author on reasonable request.

\section{Declarations}

Conflict of interest The authors declare that they have no competing interests.

Ethics approval and consent to participate Written informed consent was provided by the patient prior to the first study specific intervention. Ethical approval for study was provided by the ethics committee of Università Cattolica del Sacro Cuore, Roma (Roma PROT. N. 0033623/18 and 29042/18). The report does not present identifying images or other personal or clinical details of participants that compromise anonymity.

Consent for publication All authors reached an agreement to publish the study in this journal.

Open Access This article is licensed under a Creative Commons Attribution 4.0 International License, which permits use, sharing, adaptation, distribution and reproduction in any medium or format, as long as you give appropriate credit to the original author(s) and the source, provide a link to the Creative Commons licence, and indicate if changes were made. The images or other third party material in this article are included in the article's Creative Commons licence, unless indicated otherwise in a credit line to the material. If material is not included in the article's Creative Commons licence and your intended use is not permitted by statutory regulation or exceeds the permitted use, you will need to obtain permission directly from the copyright holder. To view a copy of this licence, visit http://creativecommons.org/licenses/by/4.0/.

\section{References}

1. Bray F, Ferlay J, Soerjomataram I, Siegel RL, Torre LA, Jemal A (2018) Global cancer statistics 2018: GLOBOCAN estimates of incidence and mortality worldwide for 36 cancers in 185 countries. CA A Cancer J Clin 68:394-424

2. Orditura M, Galizia G, Sforza V, Gambardella V, Fabozzi A, Laterza MM et al (2014) Treatment of gastric cancer. World J Gastroenterol 20:1635-1649

3. Bang YJ, Kim YW, Yang HK, Chung HC, Park YK, Lee KH et al (2012) Adjuvant capecitabine and oxaliplatin for gastric cancer after D2 gastrectomy (classic): a phase 3 open-label, randomised controlled trial. Lancet 379:315-321

4. Fuse N, Bando H, Chin K, Ito S, Yoshikawa T, Tsuburaya A et al (2017) Adjuvant capecitabine plus oxaliplatin after D2 gastrectomy in Japanese patients with gastric cancer: a phase II study. Gastric Cancer 20:332-340

5. Fuentes E, Ahmad R, Hong TS, Clark JW, Kwak EL, Rattner DW et al (2016) Adjuvant therapy completion rates in patients with gastric cancer undergoing perioperative chemotherapy versus a surgery-first approach. J Gastrointest Surg 20:172-179

6. Jin H, Zhang G, Liu X, Liu X, Chen C, Yu H et al (2013) Blood neutrophil-lymphocyte ratio predicts survival for stages III-IV gastric cancer treated with neoadjuvant chemotherapy. World J Surg Oncol 11:112

7. He Q, Li G, Ji X, Ma L, Wang X, Li Y et al (2017) Impact of the immune cell population in peripheral blood on response and survival in patients receiving neoadjuvant chemotherapy for advanced gastric cancer. Tumor Biol 5:1-8

8. Fridman WH, Galon J, Dieu-Nosjean M, Cremer I, Fisson S, Damotte D et al (2010) Immune infiltration in human cancer: prognostic significance and disease control. In: Dranoff $\mathrm{G}$ (ed) Cancer immunology and immunotherapy, current topics in microbiology and immunology, vol 344. Springer, Berlin, Heidelberg 
9. Kim JW, Nam KH, Ahn S, Park DJ, Kim H, Kim SH et al (2016) Prognostic implications of immunosuppressive protein expression in tumors as well as immune cell infiltration within the tumor microenvironment in gastric cancer. Gastric Cancer 19:42-52

10. Zhou Y, Zhu G, Lu X, Zheng KI, Wang Q, Chen J et al (2020) Identification and validation of tumour microenvironment-based immune molecular subgroups for gastric cancer: immunotherapeutic implications. Cancer Immunol Immunother 69:1057-1069

11. Kelly RJ (2017) Immunotherapy for esophageal and gastric cancer. In: Dizon DS (ed) American society of clinical oncology educational book, vol 37. pp 292-300

12. Yu Y, Ma X, Zhang Y, Zhang Y, Ying J, Zhang W et al (2019) Changes in expression of multiple checkpoint molecules and infiltration of tumor immune cells after neoadjuvant chemotherapy in gastric cancer. J Cancer 10:2754-2763

13. Li Z, Gao X, Peng X, Chen MM, Zhe Li, Wei B et al (2020) Multi-omics characterization of molecular features of gastric cancer correlated with response to neoadjuvant chemotherapy. Sci Adv 6:e4211

14. Ocana A, Nieto-Jiménez C, Pandiella A, Templeton AJ (2017) Neutrophils in cancer: prognostic role and therapeutic strategies. Mol Cancer 16:137

15. Mellor KL, Powell AGMT, Lewis WG (2018) Systematic review and meta-analysis of the prognostic significance of neutrophillymphocyte ratio (NLR) after $\mathrm{r} 0$ gastrectomy for cancer. J Gastrointest Cancer 49:237-244

16. Fukuda K, Tsujitani S, Maeta Y, Yamaguchi K, Ikeguchi M, Kaibara N (2002) The expression of RCAS1 and tumor infiltrating lymphocytes in patients with T3 gastric carcinoma. Gastric Cancer 5:220-227

17. Li F, Sun Y, Huang J, Xu W, Liu J, Yuan Z (2019) CD4/CD8 + T cells, DC subsets, Foxp3, and IDO expression are predictive indictors of gastric cancer prognosis. Cancer Med 8:7330-7344

18. Hashimoto T, Kurokawa Y, Takahashi T, Miyazaki Y, Tanaka K, Makino T et al (2019) Predictive value of MLH1 and PD-L1 expression for prognosis and response to preoperative chemotherapy in gastric cancer. Gastric Cancer 22:785-792

19. Wang Y, Zhu C, Song W, Li J, Zhao G, Cao H (2018) PD-L1 expression and CD8+ T Cell infiltration predict a favorable prognosis in advanced gastric cancer. J Immunol Res 2018:4180517

20. Strippoli A, Cocomazzi A, Basso M, Cenci T, Ricci R, Pierconti $\mathrm{F}$ et al (2020) c-MYC expression is a possible keystone in the colorectal cancer resistance to EGFR inhibitors. Cancers 12:E638

21. Rossi ED, Martini M, Capodimonti S, Cenci T, Straccia P, Angrisani B et al (2014) Analysis of immunocytochemical and molecular BRAF expression in thyroid carcinomas: a cytohistologic institutional experience. Cancer Cytopathol 122:527-535

22. Martini M, Cenci T, D’Alessandris GQ, Cesarini V, Cocomazzi A, Ricci-Vitiani L et al (2013) Epigenetic silencing of Id4 identifies a glioblastoma subgroup with a better prognosis as a consequence of an inhibition of angiogenesis. Cancer 119:1004-1012

23. Zhu Y, Sun Y, Hu S, Jiang Y, Yue J, Xue X et al (2017) Comparison of five tumor regression grading systems for gastric adenocarcinoma after neoadjuvant chemotherapy: a retrospective study of 192 cases from National Cancer Center in China. BMC Gastroenterol 17:41

24. Noble F, Lloyd MA, Turkington R, Griffiths E, O'Donovan M, O'Neill JR et al (2017) Multicentre cohort study to define and validate pathological assessment of response to neoadjuvant therapy in oesophagogastric adenocarcinoma. Br J Surg 104:1816-1828

25. Corbeau I, Jacot W, Guiu S (2020) Neutrophil to lymphocyte ratio as prognostic and predictive factor in breast cancer patients: a systematic review. Cancers 12:958

26. Nejati R, Goldstein JB, Halperin DM, Wang H, Hejazi N, Rashid A et al (2017) Prognostic significance of tumor-infiltrating lymphocytes in patients with pancreatic ductal adenocarcinoma treated with neoadjuvant chemotherapy. Pancreas 46:1180-1187

27. Li Y, Wei Y, He Q, Wang X, Fan C, Li G (2018) Clinicopathological and prognostic significance of high circulating lymphocyte ratio in patients receiving neoadjuvant chemotherapy for advanced gastric cancer. Sci Rep 8:6223

28. Taube JM, Anders RA, Young GD, Xu H, Sharma R, McMiller TL et al (2012) Colocalization of inflammatory response with $\mathrm{B} 7-\mathrm{h} 1$ expression in human melanocytic lesions supports an adaptive resistance mechanism of immune escape. Sci Trans Med 4:127ra37

29. Thompson ED, Zahurak M, Murphy A, Cornish T, Cuka N, Abdelfatah E et al (2017) Patterns of PD-L1 expression and CD8 $\mathrm{T}$ cell infiltration in gastric adenocarcinomas and associated immune stroma. Gut 66:794-801

30. Galluzzi L, Buqué A, Kepp O, Zitvogel L, Kroemer G (2015) Immunological effects of conventional chemotherapy and targeted anticancer agents. Cancer Cell 28:690-714

31. Saito R, Abe H, Kunita A, Yamashita H, Seto Y, Fukayama M (2017) Overexpression and gene amplification of PD-L1 in cancer cells and PD-L1+ immune cells in Epstein-Barr virus-associated gastric cancer: the prognostic implications. Mod Pathol 30:427-439

32. Gu L, Chen M, Guo D, Zhu H, Zhang W, Pan J et al (2017) PD-L1 and gastric cancer prognosis: a systematic review and meta-analysis. PLoS ONE 12:e0182692

33. Al-Batran SE, Homann N, Pauligk C, Goetze TO, Meiler J, Kasper $S$ et al (2019) Perioperative chemotherapy with fluorouracil plus leucovorin, oxaliplatin, and docetaxel versus fluorouracil or capecitabine plus cisplatin and epirubicin for locally advanced, resectable gastric or gastro-oesophageal junction adenocarcinoma (FLOT4): a randomised, phase 2/3 trial. Lancet 393:1948-1957

34. Wang L, Zhang Q, Ni S, Tan C, Cai X, Huang D et al (2018) Programmed death-ligand 1 expression in gastric cancer: correlation with mismatch repair deficiency and Her2-negative status. Cancer Med 7:2612-2620

35. Wang F, Liu Z, Xia Y, Zhou C, Shen X, Li X et al (2015) Changes in neutrophil/lymphocyte and platelet/lymphocyte ratios after chemotherapy correlate with chemotherapy response and prediction of prognosis in patients with unresectable gastric cancer. Oncol Lett 10:3411-3418

36. Li Z, Li S, Ying S, Zhang L, Shan F, Jia Y et al (2020) The clinical value and usage of inflammatory and nutritional markers in survival prediction for gastric cancer patients with neoadjuvant chemotherapy and D2 lymphadenectomy. Gastric Cancer 23:540-549

37. Elfving H, Mattsson JSM, Lindskog C, Backman M, Menzel U, Micke P (2019) Programmed cell death ligand 1 immunohistochemistry: a concordance study between surgical specimen, biopsy, and tissue microarray. Clin Lung Cancer 20:258-262

38. Ye M, Huang D, Zhang Q, Weng W, Tan T, Qin G et al (2020) Heterogeneous programmed death-ligand 1 expression in gastric cancer: comparison of tissue microarrays and whole sections. Cancer Cell Int 24(20):186

39. Lee JS, Won HS, Sun DS, Hong JH, Ko YH (2018) Prognostic role of tumor-infiltrating lymphocytes in gastric cancer: a systematic review and meta-analysis. Medicine (Baltimore) 97:e11769

40. Chakrabarti J, Holokai L, Syu L, Steele N, Chang J, Dlugosz A et al (2018) Mouse-derived gastric organoid and immune cell co-culture for the study of the tumor microenvironment. Methods Mol Biol 1817:157-168

Publisher's Note Springer Nature remains neutral with regard to jurisdictional claims in published maps and institutional affiliations. 


\section{Authors and Affiliations}

\section{Ina Valeria Zurlo ${ }^{1}$ Mattia Schino ${ }^{2}$ - Antonia Strippoli ${ }^{1} \cdot$ Maria Alessandra Calegari ${ }^{1}$ - Alessandra Cocomazzi ${ }^{2}$. Alessandra Cassano ${ }^{1,3}$. Carmelo Pozzo ${ }^{1,3}$ - Mariantonietta Di Salvatore ${ }^{1}$ - Riccardo Ricci ${ }^{1,2}$. Carlo Barone ${ }^{1,3}$. Emilio Bria $^{1,3} \cdot$ Giampaolo Tortora $^{1,3} \cdot$ Luigi Maria Larocca $\cdot$ Michele Basso ${ }^{1} \cdot$ Maurizio Martini ${ }^{1,2} \mathbb{D}$}

Ina Valeria Zurlo

valeriazurlo26@gmail.com

Mattia Schino

mattia.schino01@icatt.it

Antonia Strippoli

strefoantonia@gmail.com

Maria Alessandra Calegari

mariaalessandra.calegari@policlinicogemelli.it

Alessandra Cocomazzi

alessandra.cocomazzi@libero.it

Alessandra Cassano

alessandra.cassano@unicatt.it

Carmelo Pozzo

carmelo.pozzo@unicatt.it

Mariantonietta Di Salvatore

disalvatore.mariantonietta@gmail.com

Riccardo Ricci

riccardo.ricci@unicatt.it
Carlo Barone

carloantonio.barone@policlinicogemelli.it

Emilio Bria

emilio.bria@unicatt.it

Giampaolo Tortora

giampaolo.tortora@unicatt.it

Luigi Maria Larocca

luigimaria.larocca@unicatt.it

Michele Basso

michele.basso@policlinicogemelli.it

1 Fondazione Policlinico Universitario A. Gemelli IRCCS, Largo A. Gemelli 8, 00168 Rome, Italy

2 Division of Pathology, Department of Health Science and Public Health, Università Cattolica del Sacro Cuore, Largo Francesco Vito 1, 00168 Rome, Italy

3 Division of Oncology, Department of Medicine and Translational Surgery, Università Cattolica del Sacro Cuore, Largo F. Vito 1, 00168 Rome, Italy 